\title{
Large Metal Objects as Near Field UHF RFID Antennas
}

\author{
Shuai Yang ${ }^{1}$, Student Member, IEEE, Nicolas Scirocco, Student Member, IEEE, Michael Crisp, Member, IEEE, \\ Richard V. Penty, Senior Member, IEEE, and Ian H. White, Fellow, IEEE \\ Electrical Division, Department of Engineering, University of Cambridge, Cambridge, United Kingdom \\ ${ }^{1}$ sy313@cam.ac.uk
}

\begin{abstract}
This paper demonstrates that a long thin metal structure can act as a near-field antenna for a passive UHF RFID system. It is demonstrated that a conventional metal mountable tag can be detected up to $30 \mathrm{~m}$ along a metal bar from the feed point far exceeding the maximum far field free space range of the tag when mounted on metal. Further system improvements are achieved by altering the physical length of the metal structure or by changing the carrier frequency used. It is demonstrated that the fields generated along the structure (e.g. E-field or $\mathrm{H}$-field) dictate the antenna type which will be best suited for coupling to it. A conventional passive tag utilizing a dipole antenna and oriented normal to the metal surface efficiently harvests the E-field. It is deduced that the tag can be continuously detected up to at least $50 \mathrm{~m}$ along a bar from the antenna feed.
\end{abstract}

Keywords-Radio Frequency Identification; Near field; metal; configuration control; UHF; RFID; sensor.

\section{INTRODUCTION}

Radio Frequency Identification (RFID) utilizes the exchange of power and information carried by electromagnetic (EM) waves between a tag and reader to automatically identify and track objects. Over the last decade, this technology has found applications in supply chain logistics and transportation [1]. Passive UHF (860-960 MHz) RFID has enjoyed success due to potentially longer read ranges and low-cost tag manufacture compared with high frequency (HF, $13.56 \mathrm{MHz}$ ) and low frequency (LF, 134 $\mathrm{kHz}$ ) RFID systems.

A challenge for UHF RFID has been its problematic operation in metallic environments and with the tagging of metallic objects. Advances in tag antenna design have mitigated this latter issue and metal mountable tags are now commercially available for oil/gas pipelines, industrial manufacturing, and vehicle tracking [2]. Typically, the antennas for these tags use the metal object they are mounted on as a ground plane and separate the metal surface and radiating element with a dielectric material. One drawback of these tags is that their antennas are generally directional, with the peak gain normal to the surface they are mounted on. This can cause problems when the interrogator antenna is at an oblique angle of incidence to the tags.
A solution to this is to operate in the near field by bringing the RF source closer to the tag. At present the focus of research in this area has been on reader antenna design [34], or tag antenna design [5-6].

In this paper we take advantage of the conducting properties of a metal structure so that it behaves as a large near field antenna. Using a direct electrical feed in the form of a T-match connected to a metal structure we can enhance the read performance of an RFID tag as shown in Fig. 1.

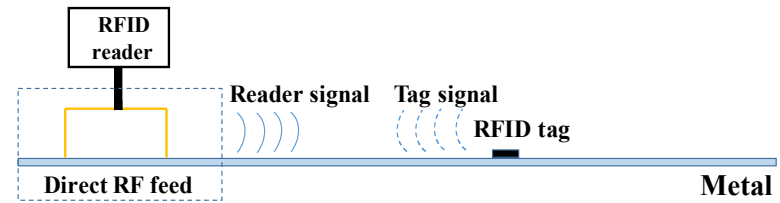

Fig. 1 Diagram of the proposed RFID system

It is shown that a long thin metal bar reduces the rate at which the reader signal falls off as the tag is moved further away from the feed point. As a result, the read range of a tag attached to the metal or positioned carefully nearby to exploit the field along the metal structure can far exceed the range in free space. From our results we believe that this technique can find many applications, such as in configuration control systems, where the reader and tags share the same metal structure. Integration with sensors may open up new fields and applications such as structural health monitoring of suspension cables.

The paper is organized as follows. The induced EM fields on metallic surfaces by a nearby antenna are introduced in Section II. Section III presents a method for direct, conducted excitation of the metal object. Sections IV and V present experimental results of a tag designed for operation on metal reading along the metal structure. Section VI proposes methods for optimizations for the system and demonstrates the read performance of a conventional passive RFID tag with a dipole antenna along the metal structure. Finally, conclusions are drawn in Section VII.

\section{RF PROPAGATION IN FREE SPACE AND ON A METALLIC SURFACE}

If a long thin metal bar is placed in front of an antenna, the 
E-field distribution above the metallic surface is distorted compared with that of free space. To visualize this distribution, a FEKO [7] simulation is carried out. In Fig. 2(a), a $5 \mathrm{~cm} \times 0.6 \mathrm{~cm} \times 3 \mathrm{~m}$ aluminum bar with conductivity of $38 \times$ $10^{6} \mathrm{~S} / \mathrm{m}$ is modelled with one end $35 \mathrm{~cm}$ away from a halfwave dipole antenna. The physical center of the dipole is offset $1 \mathrm{~cm}$ above the metal bar top face and the simulation is performed using a carrier frequency of $865.7 \mathrm{MHz}$.

A strong surface current (Fig. 2(b)) is induced on the metallic surface. Since there is no load at the bar end the current sees a sharp impedance change leading to a strong reflection of current back along the bar in addition to some radiation. The reflected current interferes with the incident current to produce a standing wave pattern. The field

(a)

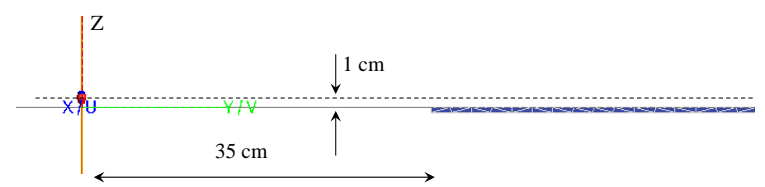

(b)
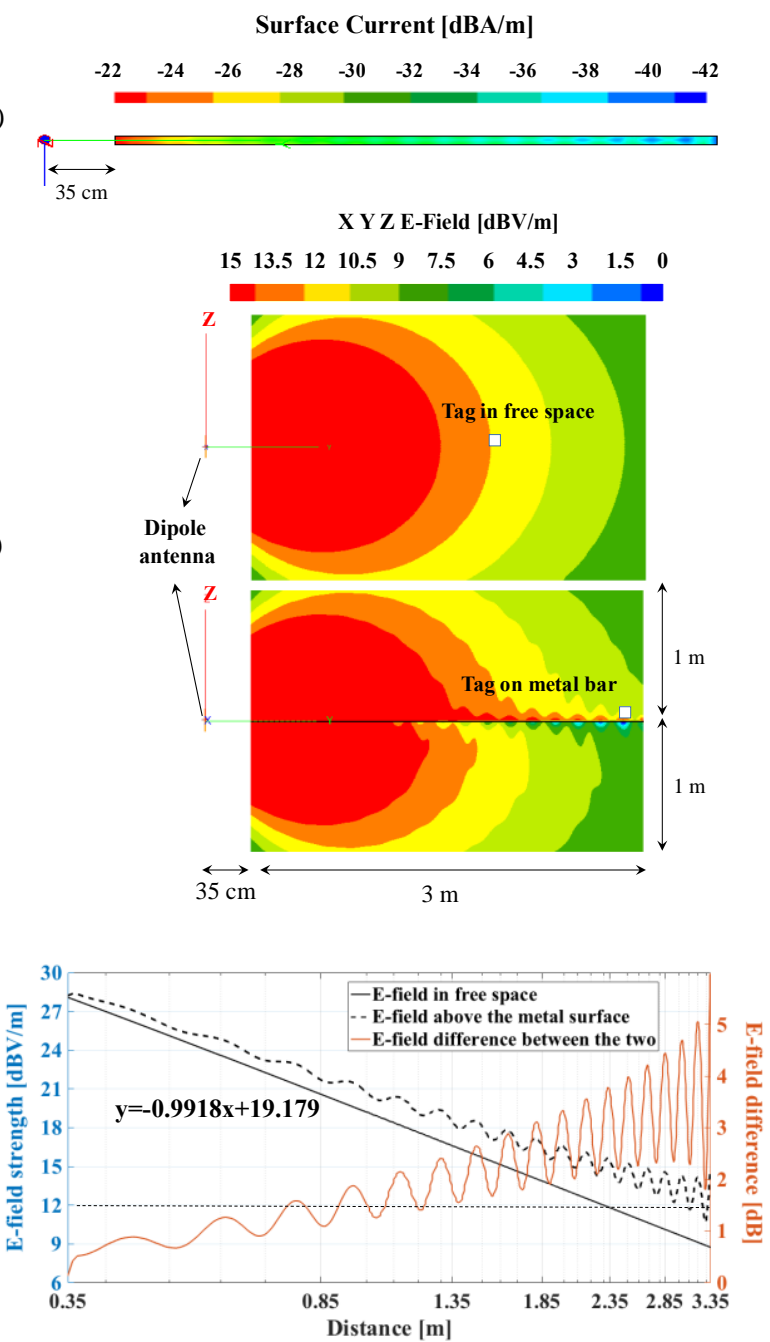

(d)

Fig. 2 (a) FEKO model (b) surface current on the metal bar (c) Efield distribution in free space and above the metal surface (d) E-field distribution as a function of distance distribution on top of the metallic surface is shown in Fig. 2(c).

Fig. 2(d) shows that in free space, the E-field strength (black line) decreases monotonically and is inversely proportional to distance. The E-field just above the top of the metallic surface is given by the black dotted line. It can be seen that its average gradient is shallower than free space, but there are ripples due to the reflections just discussed. The field strength above the bar is greater than in free space due to the surface currents induced and conducted by the bar (although below the bar the field strength is lower due to cancellation of the field arising from the induced current with that from the antenna). The orange curve shows a difference of up to $5 \mathrm{~dB}$ between the E-field strength above the bar and in free space. Due to the higher field seen above the bar, the maximum read range of a tag along the metallic surface is expected to exceed the range in free space. For example, if a tag requires a field strength of $12 \mathrm{dBV} / \mathrm{m}$ to be read in free space, then figures 2(b) and 2(c) indicate that the maximum range in free space is $2.3 \mathrm{~m}$ (illustrated by the white square); however, if read on the metal bar, the range can be extended to $2.9 \mathrm{~m}$. Beyond $2.9 \mathrm{~m}$ reading is still possible at some locations, but reflections from the bar end cause deep nulls in the field strength. Therefore it will not be read at all points beyond this distance.

\section{IMPEDANCE MATCHING NETWORK DESIGN}

The FEKO simulation suggests that placing the bar close to the antenna is necessary to ensure a strong field distribution above it. However, the antenna pattern is also distorted. If the objective is to read tags on the bar, a large proportion of power is wasted. A better solution would be to connect the RF antenna feed directly to the metal bar, thus making it the antenna. In this case the metal bar can be viewed as an electrically long dipole, with the tag in its near field.

\section{A. Impedance Measurement}

An impedance matching network is necessary to maximize power transfer to the metal bar. Fig. 3 shows an antenna feed comprising a T-match [8] made of copper wires connected directly to the bar. The aluminum bar has the same dimensions the previous EM model. The distances from the left feed point to the left end of the bar is $13 \mathrm{~cm}$, and the feed point separation is $17.5 \mathrm{~cm}$.

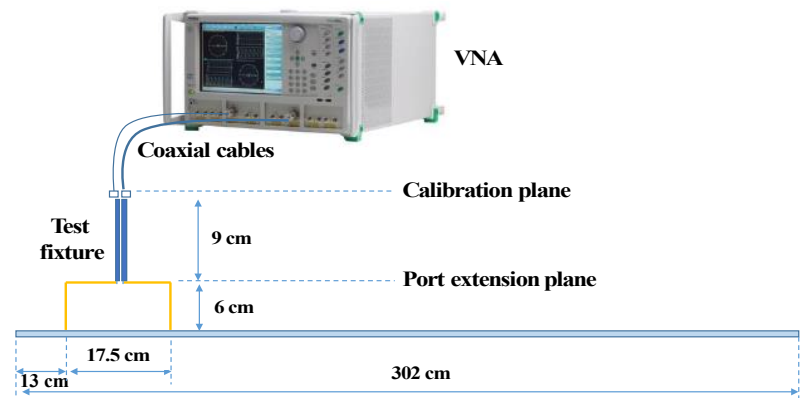

Fig. 3 Impedance measurement setup

Since the T-match requires a balanced feed, the method in [9] is used to determine the impedance using a 2-port Vector Network Analyser (VNA). The experimental impedance 


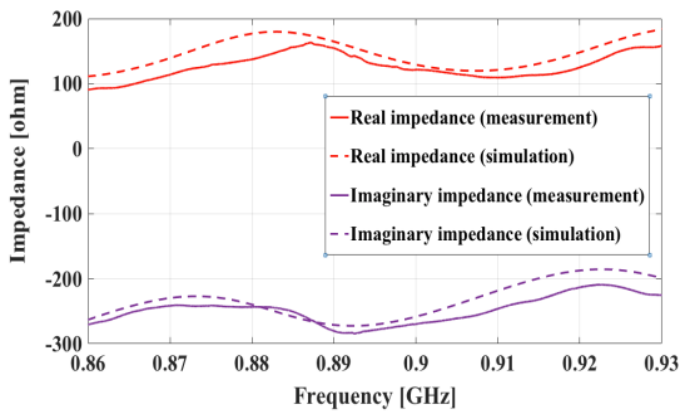

Fig. 4 Simulated and measured impedance

measurements are shown in Fig. 4 along with a FEKO simulation. The measured impedance values are in general agreement with the simulation with the deviations between the two possibly caused by (a) errors introduced by the test fixture port extension method; and (b) nearby objects which may produce loading effects that influence the antenna impedance.

\section{B. Matching Network Design}

A balun is inserted between the coaxial cable and the Tmatch feed to allow connection to unbalanced co-axial cables. Based on the manufacturer's S-parameters for the balun and the measured impedance of the metal bar, a simple L-match network is designed (Fig. 5(a)). The system return losses with and without the matching network are shown in Fig. 5(b).
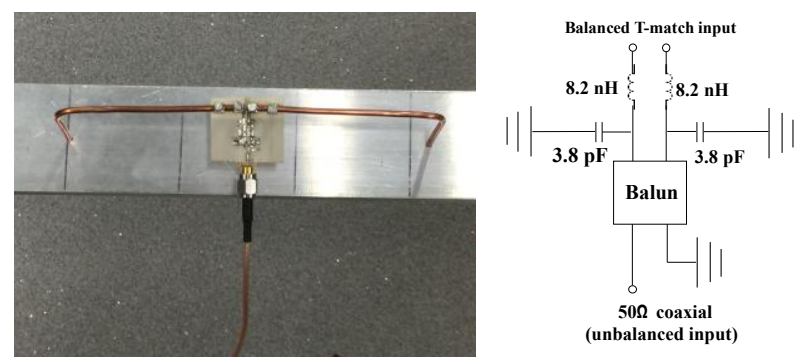

(a)

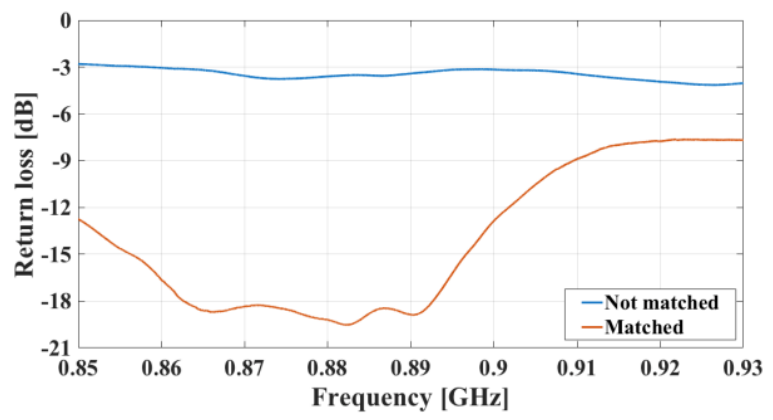

(b)

Fig. 5 (a) Schematic of the impedance matching network (b) Return loss before and after impedance matching

The return loss is improved from $-3 \mathrm{~dB}$ to $-18 \mathrm{~dB}$ in the desired frequency band (865-868 MHz). The achieved return loss is comparable to a typical RFID patch antenna. Low return loss is important for the proposed RFID system due to the self jamming effect desensitizing the receiver [10].

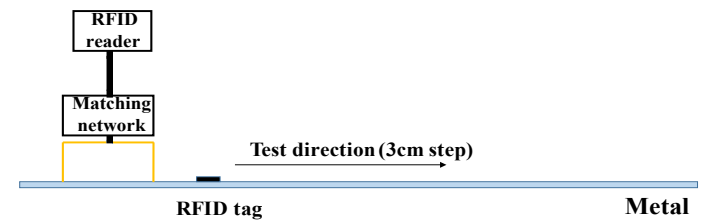

Fig. 6 Experimental setup to determine tag read performance along the bar

\section{DEMONSTRATION OF TAG READING ALONG THE BAR}

Fig. 6 illustrates the experimental setup to investigate the required conducted electrical power to read a tag on the metal bar. An Irontag Aero [11] using an Impinj Monza-8K IC [12] is selected as the measurement tag. The tag is designed for long range operation in the far field when attached to a metal object.

An Impinj Speedway R420 [12] RFID reader with a fixed frequency of $865.7 \mathrm{MHz}$ is used as the interrogator. The tag is moved towards the bar end in $3 \mathrm{~cm}$ steps and the tag threshold (the lowest conducted power allowing successful identification) recorded at each location. The results are plotted in Fig. 7. Note that the y-axis has been reversed to allow direct comparison between the required reader output power at threshold with later simulation results where field strength is calculated for a fixed conducted RF power.

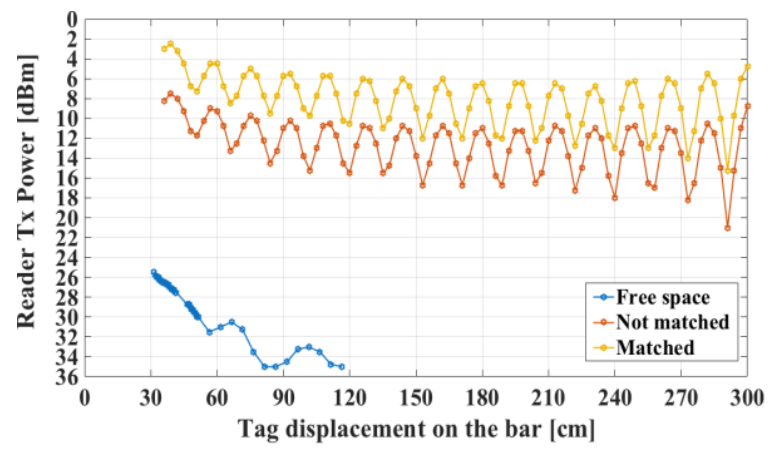

Fig. 7 Tag threshold distribution along the bar and in free space

The overall trend shows increasing required transmit power as the distance away from the feed point increases. Superimposed on this trend is a strong ripple with a characteristic wavelength of $\sim 17 \mathrm{~cm}$ (approximately $\lambda / 2$ of the carrier in free space, $\sim 17.4 \mathrm{~cm}$ ). The first minimum is located $9 \mathrm{~cm}$ away from the bar end which is approximately a $\lambda / 4$ $(8.7 \mathrm{~cm})$. This power distribution is similar to the field distribution on an off-center fed long (multi-wavelength) dipole.

The blue curve shows the free space threshold power for the tag attached to a small metal plate. The same tag is used, but a standard $7 \mathrm{dBi}$ patch antenna is substituted for the feed and bar. The test is not performed in an anechoic chamber and as a result the tag threshold does not decrease monotonically as the distance from the reader antenna increases as a result of multipath effects. The maximum transmit power used is 35 $\mathrm{dBm}$ EIRP as per local regulation resulting in a maximum free space range of only $1.2 \mathrm{~m}$. In comparison, when using the 
metal bar with a direct feed, $20 \mathrm{~dB}$ less power is required at this distance. At the maximum range recorded, i.e. $2.7 \mathrm{~m}$ away from the T-match feed, limited by the length of the bar, less than $21 \mathrm{dBm}$ is required to read the tag showing the potential to read over even longer distances.

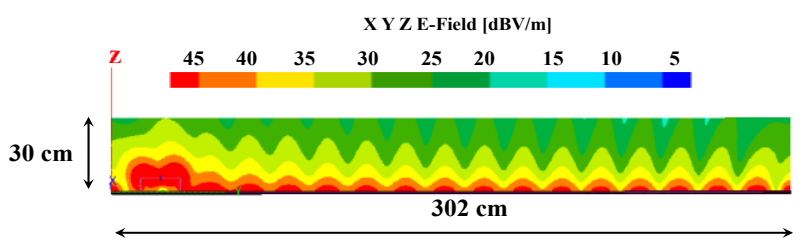

(a)

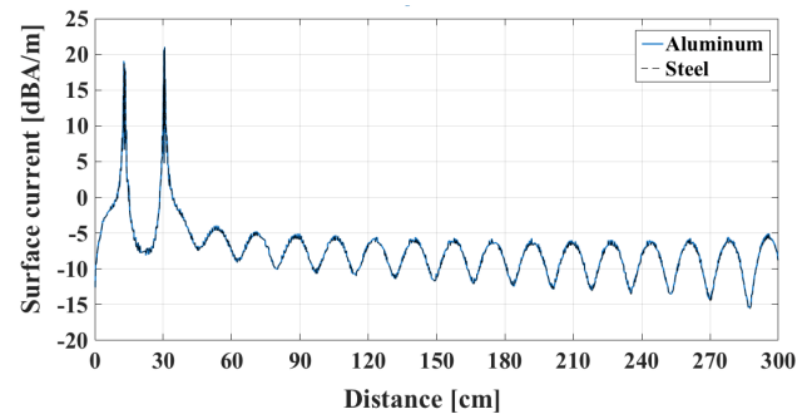

(b)

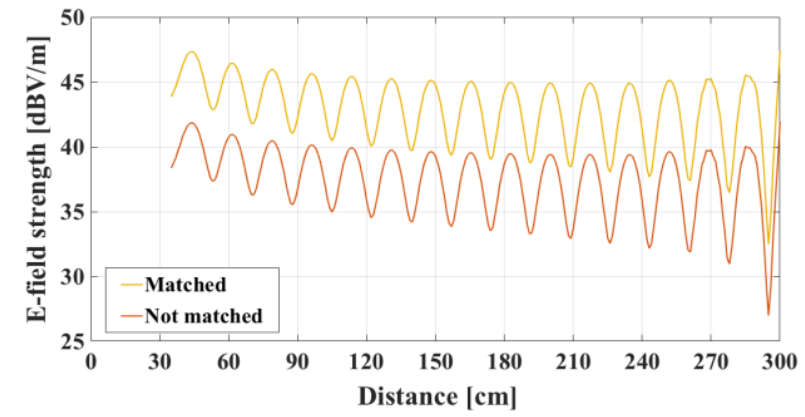

(c)

Fig. 8 (a) E-field distribution up to $30 \mathrm{~cm}$ above the bar surface (b) Surface current distribution along the centre line of the metal bar (current density of a less conductive material, carbon steel, is also plotted for comparison) and (c) E-field distributions $5 \mathrm{~mm}$ above the aluminum bar

Fig. 8 shows the E-field distributions up to $30 \mathrm{~cm}$ above the metal bar generated by a FEKO model. It can be seen from Fig. 8(a) that, a large portion of field is confined close to the metal surface (the E-field drops by almost $20 \mathrm{~dB}$ from $0 \mathrm{~cm}$ to $30 \mathrm{~cm}$ away from the surface). The rapid change of field strength with distance is characteristic of near field operation. In addition, the surface current density along the center line of the bar (Fig. 8(b)) is strongest close to the contact points of the T-match, reducing with increasing distance along the bar away from the feeds owing to radiative losses. Also plotted are the surface currents when a less conductive carbon steel $\left(6 \times 10^{6}\right.$ $\mathrm{S} / \mathrm{m}$, with a relative permeability of 100 ) is used. Here the simulation results show that the overall antenna efficiency degrades from $99.8 \%$ to $97.8 \%$ as a result of the 12 times increase in ohmic losses (from $1.76 \mathrm{~mW}$ to $22 \mathrm{~mW}$, with $1 \mathrm{~W}$ fed to the metal structure). However, since the ohmic loss is a small proportion of the overall losses, no significant change in surface current density can be seen between the two materials in Fig. 8(b).

Fig. 8(c) shows the E-field distribution $5 \mathrm{~mm}$ above the metal surface. To allow direct comparison with the measured results, a virtual port is configured as the conjugated match of the simulated metal bar and feed. The trends are similar to those seen in Fig. 7. However, there is a constant $5 \mathrm{~cm}$ offset in the locations of the maxima and minima of the ripple between the experimentally measured threshold power and the simulated E-field. This could be because (i) the $3 \mathrm{~cm}$ experimental resolution step is too large to precisely locate the maxima and minima and (ii) the tag is not electrically centered about its physical center (which is used as the measured location for the experimental results). It is also possible that differences between the simulated and experimental impedance matches will introduce small phase offsets.

To study how the tag power threshold varies normal to the metal surface, the tag is placed one meter away from the left bar end and its threshold is measured as it is moved in the vertical direction in increments of $1 \mathrm{~cm}$ away from the metal surface (in this case the tag is no longer mounted on metal as the separation increases). The measurement results are shown in Fig. 9.

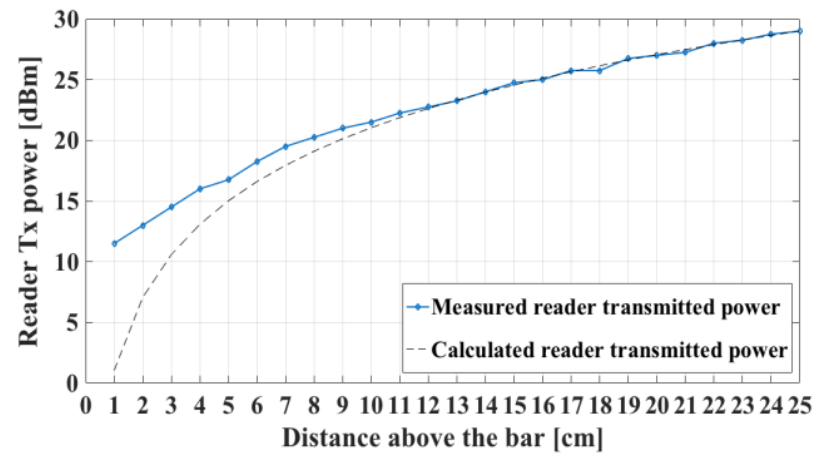

Fig. 9 Minimum reader power requirement of the tag above the bar. The dotted line calculates the powers using $1 / D^{2}$ dependence and measured power at $25 \mathrm{~cm}$ as the reference point

It is difficult to determine the phase center (the effective far field origin of the radiation) of the whole metal structure due to its large dimensions. However, one can easily see from Fig. 9 that an obvious transition in the behavior occurs $13 \mathrm{~cm}$ above the metal surface where the system behavior transitions from near field to far field (i.e. $1 / r^{2}$ ). Since the thickness of the tag is only $5 \mathrm{~mm}$, the tag operates in the near-field of the antenna. (It should be noted that the tag is likely to become less sensitive with increasing distance from the metal surface since it is designed for operation on a metal surface)

\section{MAXIMUM SYSTEM RANGE ESTIMATION}

To determine experimentally the ultimate limits on read range by using a longer bar is not possible given equipment availability. Instead we modify our simulation by extending the bar length up to $50 \mathrm{~m}$. The resulting distributions of the Efield strength $5 \mathrm{~mm}$ above the bar with a $1 \mathrm{~W}$ source power are shown in Fig. 10. 


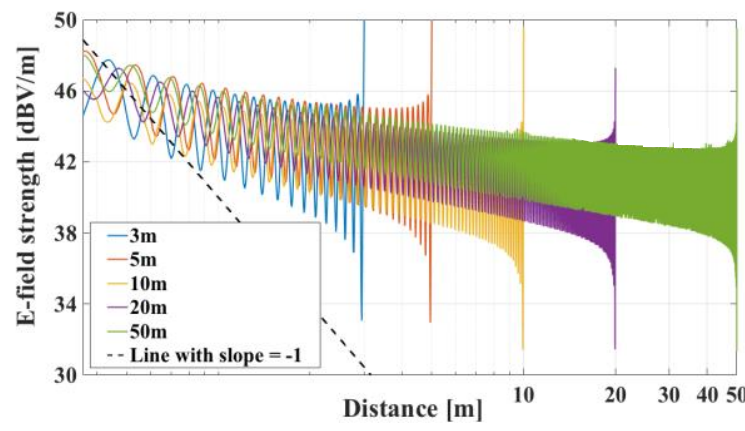

Fig. 10 E-field distribution $5 \mathrm{~mm}$ above the bar with different length

Both axes in Fig. 10 are plotted on a logarithmic scale and the black dotted line represents the E-field distribution which is inversely proportional to distance (i.e. E-field distribution in free space). Comparing the E-field in free space with that above the metal surface, free space has the expected $20 \mathrm{~dB} /$ decade reduction with distance while above the bar the field does not follow a constant slope, but over the range of interest only falls a few $\mathrm{dBs}$ per decade. It is worth noting that along the bar there is significant ripple in the E-field The ripple amplitude also increases as the direct and reflected wave amplitudes become comparable with increasing distance from the feed. This results in deeper nulls close of the far end of the bar. Close to the feed point, as the bar length increases, the influence of the reflected waves becomes weaker, yielding a smaller amplitude ripple.

\begin{tabular}{|c|c|c|c|c|c|c|}
\hline $\begin{array}{l}\text { Bar } \\
\text { length } \\
{[\mathrm{m}]}\end{array}$ & $\begin{array}{c}\text { Simulated } \\
\text { gain } \\
{[\mathrm{dBi}]}\end{array}$ & $\begin{array}{c}\text { Allowable } \\
\text { conducted } \\
\text { power } \\
{[\mathrm{dBm}]}\end{array}$ & $\begin{array}{c}\text { E-field peak with } \\
1 \mathrm{~W} \text { input } \\
{[\mathrm{dBV} / \mathrm{m}]}\end{array}$ & $\begin{array}{c}\text { E-field peak at } \\
\text { allowable } \\
\text { conducted } \\
\text { power } \\
\text { [dBV/m] } \\
\end{array}$ & $\begin{array}{c}\text { E-field trough } \\
\text { with } 1 \mathrm{~W} \text { input } \\
\text { [dBV/m] }\end{array}$ & $\begin{array}{c}\text { E-field trough } \\
\text { at allowable } \\
\text { conducted } \\
\text { power } \\
\text { [dBV/m] }\end{array}$ \\
\hline 3 & 11.5 & 20.7 & 44.6 & 34.3 & 34.9 & 25.6 \\
\hline 5 & 12.4 & 19.8 & 45.7 & 33.5 & 34.7 & 24.5 \\
\hline 10 & 14.7 & 17.5 & 44.2 & 29.8 & 31.1 & 20.6 \\
\hline 20 & 16.1 & 16.1 & 44.2 & 28.3 & 33 & 19.1 \\
\hline $\mathbf{5 0}$ & 19.5 & 12.7 & 44.2 & 24.9 & 33 & 15.7 \\
\hline
\end{tabular}

(a)

\begin{tabular}{|c|c|c|c|c|c|c|}
\hline $\begin{array}{c}\text { Sampled } \\
\text { location } \\
\text { [m] }\end{array}$ & $\begin{array}{c}\text { Simulated } \\
\text { gain } \\
{[\mathrm{dBi}]}\end{array}$ & $\begin{array}{c}\text { Allowable } \\
\text { conducted } \\
\text { power } \\
{[\mathrm{dBm}]}\end{array}$ & $\begin{array}{c}\text { E-field peak } \\
\text { with } 1 \mathrm{~W} \text { input } \\
{[\mathrm{dBV} / \mathrm{m}]}\end{array}$ & $\begin{array}{c}\text { E-field peak at } \\
\text { allowable } \\
\text { conducted } \\
\text { power } \\
\text { [dBV/m] } \\
\end{array}$ & $\begin{array}{c}\text { E-field trough } \\
\text { with } 1 \mathrm{~W} \text { input } \\
{[\mathrm{dBV} / \mathrm{m}]}\end{array}$ & $\begin{array}{c}\text { E-field trough } \\
\text { at allowable } \\
\text { conducted } \\
\text { power } \\
\text { [dBV/m] }\end{array}$ \\
\hline 3 & \multirow{12}{*}{19.5} & \multirow{12}{*}{12.7} & 44.7 & 27.4 & 41.7 & 24.4 \\
\hline 4 & & & 44.6 & 27.2 & 41.2 & 23.9 \\
\hline 5 & & & 44.2 & 26.9 & 40.8 & 23.5 \\
\hline 6 & & & 44 & 26.7 & 40.5 & 23.1 \\
\hline 7 & & & 43.9 & 26.6 & 40.3 & 23 \\
\hline 8 & & & 43.7 & 26.4 & 40.18 & 22.8 \\
\hline 9 & & & 43.6 & 26.3 & 40 & 22.7 \\
\hline 10 & & & 43.5 & 26.2 & 39.9 & 22.6 \\
\hline 15 & & & 43.2 & 25.9 & 39.4 & 22 \\
\hline 20 & & & 43 & 25.7 & 38.9 & 21.6 \\
\hline 30 & & & 42.8 & 25.5 & 38.5 & 21.1 \\
\hline 40 & & & 42.7 & 25.4 & 37.8 & 20.5 \\
\hline
\end{tabular}

(b)

Table 1. Simulated gain and E-field strength with respect to different bar lengths. The allowable conducted power has taken the gain into account. (a) E-field strengths shown are for the penultimate trough and peak at the end of each bar with different length. (b) Efield strengths are shown for the nearest peak and trough to the specified location with a $50 \mathrm{~m}$ bar. Values in red highlight that the E-field strength along the corresponding bar length is higher than the tag threshold of $21 \mathrm{dBV} / \mathrm{m}$
To determine the maximum range, one must consider the limits on the allowable conducted power at the feed point. Since some RF power is lost through radiation, it is necessary to apply the regulatory limits for radiated power which in ETSI regions is $2 \mathrm{~W}$ ERP [13]. To determine the corresponding conducted power for the metal bar, the far-field gain of different bar lengths is determined by simulation and the results are shown in Table 1. The minimum E-field is also given for a $1 \mathrm{~W}$ input power at the RF feed, excluding the deepest minima $\lambda / 4$ from the end.

By comparing the experimental data with the simulation data in figures 7 and 8(c) above, it is possible to determine the relationship between the required reader transmitted power needed to achieve threshold power at a tag at a certain range and the corresponding simulated E-field strength, as shown in Fig. 11. From this it can be estimated that the threshold field for the tag is $21 \mathrm{dBV} / \mathrm{m}$.

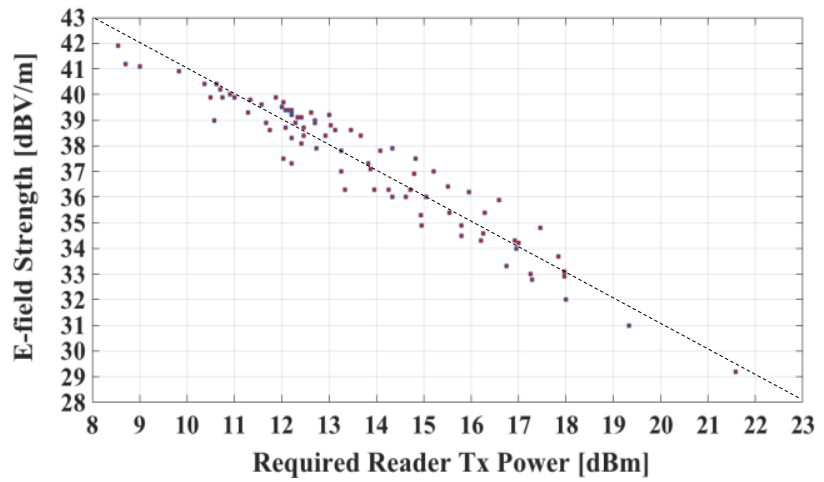

Fig. 11 Required reader transmitted power and the corresponding simulated E-field strength based on $1 \mathrm{~W}$ feed power

Based on Table 1(a), it is expected the tag would be readable anywhere on a bar with a length of $5 \mathrm{~m}(24.5 \mathrm{dBV} / \mathrm{m}$ highlighted in Table 1(a) is higher than $21 \mathrm{dBV} / \mathrm{m}$ ). For lengths greater than $5 \mathrm{~m}$ there will be E-field minima less than $21 \mathrm{dBV} / \mathrm{m}$ resulting in locations where the tag would not be read. However, more than a $50 \mathrm{~m}$ range can also be achieved if the tag is located in the E-field maxima.

Table 1(b) considers the case of a $50 \mathrm{~m}$ long bar, the Efield minima over various distances is found. It can be seen that the tag can be read continuously over the first $30 \mathrm{~m}$.

It should be noted that no optimization of the tag has been carried out for this read configuration, so longer ranges can be expected. It is also possible that the actual gain of the bar will be lower in practice than in simulation so higher conducted power could be used without exceeding regulations.

\section{SYSTEM OPTIMISATIONS}

\section{A. Major Components of the Electric and Magnetic Fields}

An important characteristic of the system is to understand which components of the electric and magnetic fields contribute to the threshold power recorded at the tag. Maxwell's equations and corresponding boundary conditions at an air-metal (perfect electric conductor (PEC)) interface predict that the electric field normal to the metal surface and 


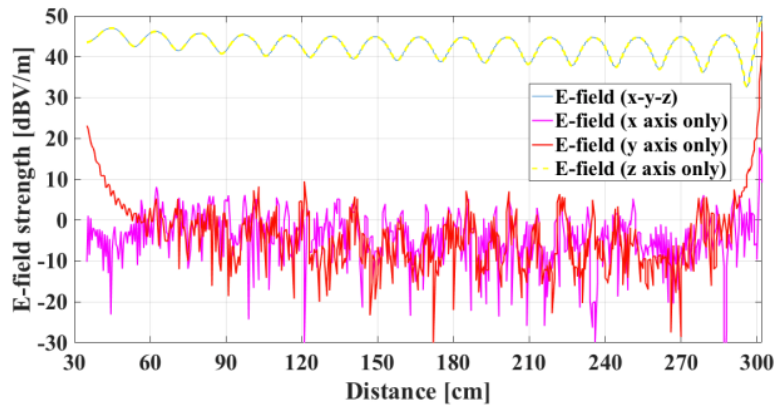

(a)

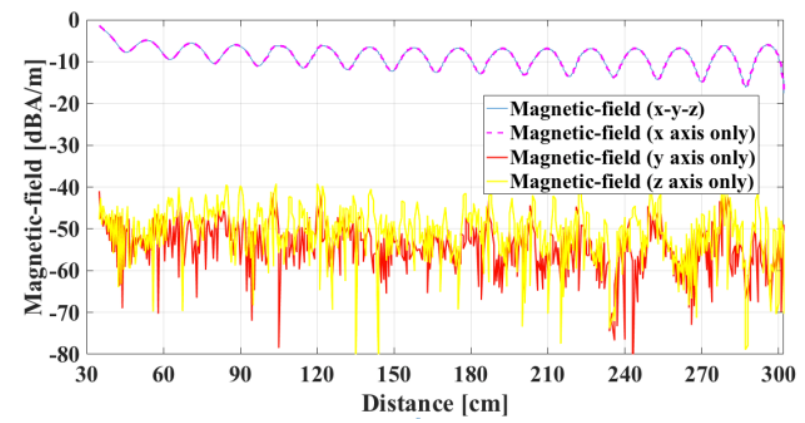

(b)

Fig. 12 (a) Electric field distribution along the aluminum bar (b) Magnetic field distribution along the aluminum bar. X-axis and $\mathrm{Y}$ axis follow the length and width of the bar respectively; $\mathrm{Z}$-axis is in the direction perpendicular to the bar surface

the magnetic field tangential to the surface are the only available field components [14]. It is expected that when the metal is less perfect (e.g. finite conductivity, rough surface) the field distributions will be deformed but the overall trend will remain. To illustrate this (Figs. 12(a) and 12(b)) a FEKO simulation of the experiment is performed showing the near fields $5 \mathrm{~mm}$ above the top of the aluminium bar. Note that the Z-component (yellow line) is perpendicular to the bar surface, the Y-component (red) is in the direction of the length of the bar and the X-component (purple) is across the width of the bar. It can be seen that the dominant component is normal to the surface in the Z-direction.

In a similar fashion for the magnetic field in Fig. 12(b) the largest contributing component is in the $\mathrm{X}$ direction tangential to the surface. Both dominant fields are perpendicular to each other and the plane they form is normal to the direction along the bar, which represents the dominant direction of surface current propagation.

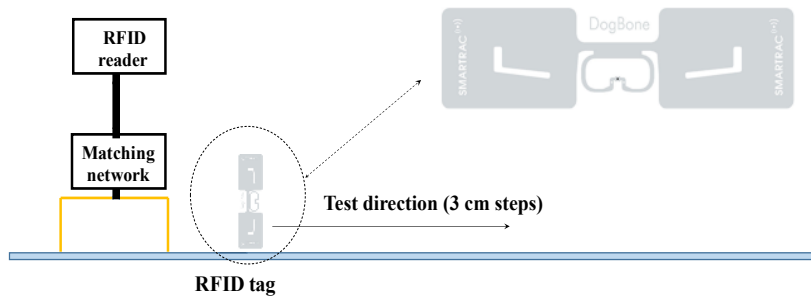

Fig. 13 Experimental setup to investigate the read performance of a dipole based tag along the bar. The inner resonating LC loop is used to improve the bandwidth of the antenna such that the tag can operate globally
One can see that the magnetic field which follows the surface current distribution (Fig. 8 (b)) along the bar having minima at the bar end whereas the electric field, which follows the voltage distribution, has a maxima. In addition, it can be seen that the E-field has its first minima $\lambda / 4$ away from the bar end whereas the magnetic field has its first maxima. The distance between each successive peak is $\lambda / 2$ of the carrier signal and thus because the two fields are in antiphase the actual phase difference between the two fields is $\lambda / 4$ or $90^{\circ}$. This is as expected for systems operating in the near field.

The metal tag under test in Section $\mathrm{V}$ has a loop-less patch antenna which is designed to harvest the far-field E-field. Based on the distributions of the tag threshold and simulated electric and magnetic fields at each test location, one can deduce that it is the enhanced E-field that helps improve the range of the metal tag along the bar. We can extend the use of the approach to optimize the orientation of generic RFID tags once we know which antennas they utilize.

\section{B. Read Performance of a Passive tag with a dipole antenna}

To determine if the approach can also be used to allow standard tags to be read along a bar, as shown in Fig. 13, a dipole based passive tag (DogBone [15]) is placed vertically and moved towards the bar end in $3 \mathrm{~cm}$ steps. The recorded tag thresholds at each test location are plotted in Fig. 14.

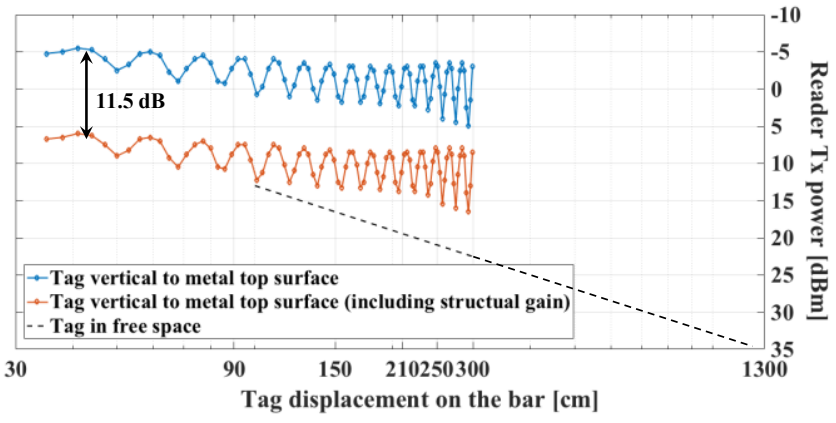

Fig. 14 A dipole-antenna-based passive tag's threshold distribution along the metal bar. The tag is placed vertical to the metal's top surface to harvest the E-field

The vertical configuration of the dipole antenna allows the normal E-field above the metal surface to induce a potential difference between the dipole's two arms and hence drive the tag IC. As shown in Fig. 14, the passive tag's threshold distribution is very similar to that of the metal tag (Fig. 7), except that, the dipole antenna requires roughly $10 \mathrm{~dB}$ less power than its metal mountable counterpart. The contribution due to the different tag ICs is negligible since both have similar sensitivity (around $-19 \mathrm{dBm}$ ). Up to $5 \mathrm{dBm}$ reader transmitted power is needed to read the tag anywhere along the $3 \mathrm{~m}$ bar, the maximum transmission loss can be estimated as around $24 \mathrm{~dB}$ (i.e. $5 \mathrm{~dB}$ minus the IC's sensitivity of -19 $\mathrm{dBm})$, which is much less than that of the metal tag ( $34 \mathrm{~dB})$.

To determine if there is any range improvement compared to free space, the tag's threshold at $1 \mathrm{~m}$ away from a far-field antenna is also measured and found to be $13 \mathrm{dBm}$. Based on this value and the Friis transmission equation in free space, tag thresholds at further distances are calculated, as shown by the 


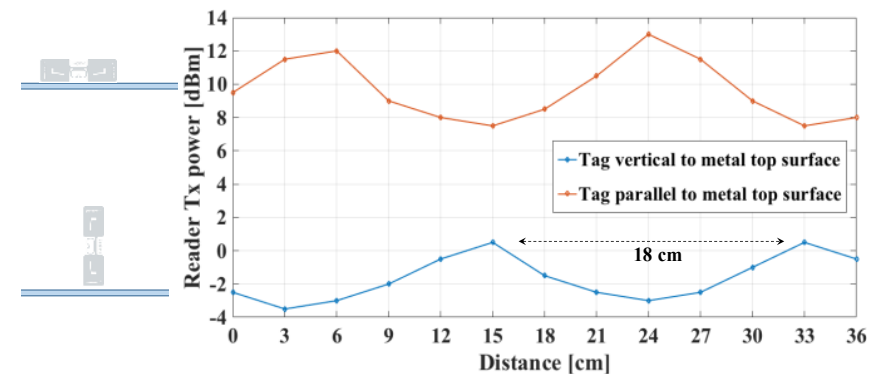

Fig. 15 Tag thresholds along a $36 \mathrm{~cm}$ segment picked from the long bar. The threshold distribution follows the E-field distribution when the antenna is vertical to the metal surface. When the tag is horizontally placed with the plane of the inner loop vertical to the metal surface, the tag threshold follows the magnetic-field distribution. Both distributions have a characteristic wavelength of 18 $\mathrm{cm}$ which is around a half wavelength of the carrier signal

black dotted curve in Fig. 14. As can be seen, the maximum range of the tag in free space is around $12.5 \mathrm{~m}$ (i.e. at the maximum allowable reader transmitted power of $35 \mathrm{dBm}$ EIRP [13]). However, when read on the metal bar, the dipole tag requires much less power compared to free space, even when taking the $11.5 \mathrm{dBi}$ simulated structural gain (see Table 1) into account. If we assume that the tag requires an E-field of $11 \mathrm{dBV} / \mathrm{m}$ to operate $(10 \mathrm{~dB}$ more sensitive than the metal tag according to Figs. 7 and 11), then according to Table 1, the tag is expected to be read continuously along a bar of at least $50 \mathrm{~m}$ length.

To date, there have been various forms of the dipole antenna designed for passive UHF RFID tags, such as meander and bowtie antennas. Most of them have a small LC loop inserted between the dipole and the tag IC to provide and impedance match with sufficient bandwidth for the tag to operate globally $(860-960 \mathrm{MHz})$ [16]. For near-field operation, this small LC loop can be used to harvest the magnetic field. However, the tag must be orientated correctly so that the magnetic flux can pass through the loop and induce current in the IC. As shown in Fig. 15, the passive tag is orientated horizontally but with the plane of the LC loop vertical to the metal surface. It can be seen from the red curve that the tag is still detectable, but the required reader transmitted power at each test location is much higher than that of the tag when it is placed vertically on the metal bar (blue curve). This is mainly due to the weak magnetic field produced by the metal bar and small loop. In addition, the two distributions are out of phase by $90^{\circ}$ as expected

\section{Methods to Shift the E-field Distribution}

Based on the results demonstrated so far, it is shown that the E-field troughs above the bar limit the system's operating range. The most obvious way to improve the system's performance is to eliminate these E-field ripples by terminating the bar end with a proper load This is common practice for travelling wave antennae [17]. However, such an approach would also lose the benefits of the lower tag thresholds resulting from the E-field peaks which can be up to $3 \mathrm{~dB}$. An alternative solution is shift the E-field distribution left or right until the initial E-field distribution is completely reversed with the nulls becoming peaks.

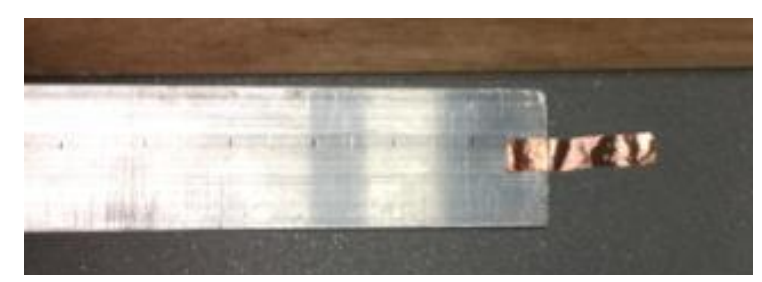

(a)

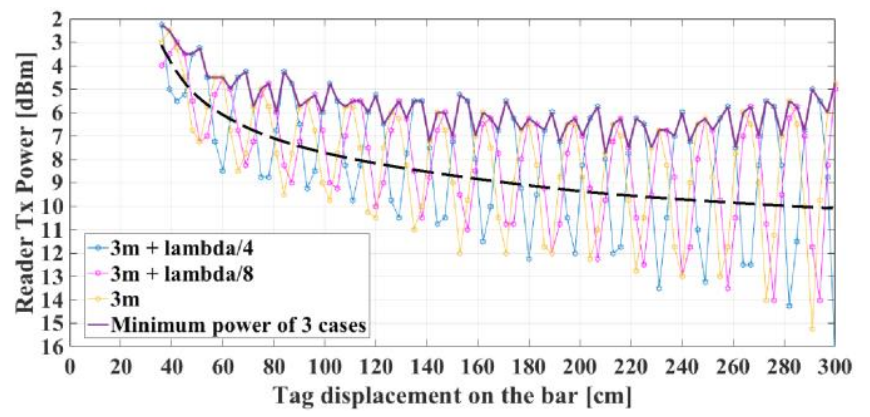

(b)

Fig. 16 (a) A sticky copper tape is attached to the end of the bar to physically extend its length (b) tag threshold distribution along the bar with different lengths

\section{i.Antenna Lengthening}

Fig. 16(a) shows the experimental setup to physically change the electrical length of the metal bar by attaching copper tape. Since no significant change was found in the return loss of the RF feed, the RF feed was unchanged in the tests. The results are shown in Fig. 16(b).

It can be observed from the yellow curve that for the original bar, the first threshold trough is located $9 \mathrm{~cm}$ from the bar end, which is around a $\lambda / 4$ at $865.7 \mathrm{MHz}$. However, when the bar is extended by $\lambda / 8(\sim 4.3 \mathrm{~cm})$, the overall distribution is shifted rightwards, and the first threshold trough occurs around $6 \mathrm{~cm}$ away from the bar end (excluding the copper tape). When the bar is further lengthened to $\lambda / 4(\sim 8.7 \mathrm{~cm})$, as shown by the blue curve, the tag threshold distribution is completely reversed with the first trough occurring at the original bar end. In such a configuration, the locations that initially required higher reader transmitted powers have lower thresholds. However, the opposite is also true, i.e. higher reader transmitted powers are now needed for those locations which initially required less reader transmitted powers.

In practice physically changing the bar length is not likely to be practical, however, the electrical lengthen could be altered by inserting an inductor or a capacitor in series with it instead [18].

\section{ii. Frequency Hopping}

Another method to shift the current (and hence field) distribution on the bar is to change the carrier frequency. Fig. 17 shows the tag threshold distribution for an $18 \mathrm{~cm}$ segment of the bar, when the carrier frequency is changed within the lower ETSI band (i.e. 865.7-867.5 MHz), and the upper ETSI band (i.e. 916.3-919.9 MHz). Due to the narrow bandwidth, only $1 \mathrm{~dB}$ improvement can be seen when the frequency is switched from 865.7 - 867.5 MHz, or from 916.3 - 919.9 MHz. However, if we switch the carrier frequency between 


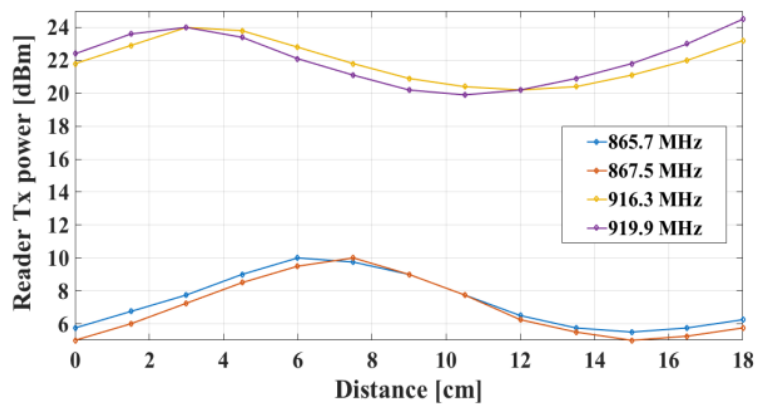

Fig. 17 Tag threshold distribution along the bar with different carrier frequency. The higher reader transmitted power requirements at $916.3 \mathrm{MHz}$ and $919.9 \mathrm{MHz}$ are partly due to the higher return loss of the reader RF feed at these frequencies (see Fig. 5 (b))

865.7 MHz and 919.9 MHz, (allowable under the new ETSI RFID standard [18]), a clear shift in the tag threshold distribution can be found. However, to make such a system work optimally, a wider-band impedance matching network for the reader RF feed is needed. The mismatch of the feed network in the upper band is the cause of the higher conducted power required in Figure 17.

\section{CONCLUSIONS}

The read performance in the UHF band of a metal tag mounted along a long thin aluminum bar which is directly driven by an RFID reader has been studied. The measurement results indicate that the system has an enhanced range compared to the same tag operating in free space. Based on simulations of system far-field gain and taking local ERP limits into consideration, the proposed system is expected to be functional at over $30 \mathrm{~m}$ with a bar length of $50 \mathrm{~m}$. Further, with optimization of the orientation of a standard RFID tag, operation in excess of $50 \mathrm{~m}$ should be possible.

Two methods to shift the electric field, and hence the tag threshold distribution along the bar have been demonstrated giving potential to further improve the read range.

This proof of concept study has focused on a long thin bar, and other geometries are likely to have less favorable properties as the surface current will spread in two directions. However, given the large link margin achieved and likely low gain (and hence high allowable conducted power) of other geometries this suggests the system may find wide ranging practical applications.

\section{ACKNOWLEDGMENT}

This work is partially supported by Innovate UK through the LAGEMOSYS project. Additional data related to this publication is available at the University of Cambridge data repository https://doi.org/10.17863/CAM.13032

\section{REFERENCES}

[1] K. Finkenzeller, RFID handbook. Chichester, England: Wiley, 2003.

[2] Xerafy. Available: www.xerafy.com [Accessed: Dec. 10 2016].

[3] D. M. Dobkin, S. M. Weigand and N. Iyer, "Segmented Magnetic Antennas for Near Field UHF RFID," Microwave Jounal, vol. 50, pp. 96-102, 2007.
[4] X. M. Qing, C. K. Goh and Z. N. Chen, "A Broadband UHF Near Field RFID Antenna," IEEE Transactions on Antennas and Propagation, vol. 58, pp. 3829-3838, Dec. 2010.

[5] K. Jaakkola and P. Koivu, "Low-cost and Low-profile Near Field UHF RFID Transponder for Tagging Batteries and Other Metal Objects," IEEE Transactionson Antennas and Propagation, pp. 692-702, Dec. 2014.

[6] M. Dhaouadi, M. Mabrouk, T. P. Vuong, A. C. D. Souza and A. Ghazel, "UHF Tag Antenna for Near Field RFID Applications," in IEEE Conference on Wireless and Microwave Technology, June 2014.

[7] FEKO. Available: www.feko.info [Accessed: Dec. 10 2016].

[8] W. L. Stutzman and G. A. Thiele, Antenna Theory and Design, John Wiley \& Sons, 1981.

[9] R. Meys and F. Janssens, "Measuring the Impedance of Balanced antennas by an S-parameter Method," IEEE Antennas and Propagation Magzine, vol. 40, pp. 62-65, Dec. 1998.

[10] M. Bolic, D. S. Ryl and I. Stojmenovic, RFID Systems: Research Trends and Challenges, Wiley, 2010.

[11] STiD. Availabe: www.stid.com [Accessed: Dec. 10 2016].

[12] Impinj. Available: www.impinj.com [Accessed: Dec. 10 2016].

[13] Electromagnetic compatibility and Radio spectrum Matters (ERM); Radio Frequency Identification Equipment operating in the band 865 $\mathrm{MHz}$ to $868 \mathrm{MHz}$ with power levels up to $2 \mathrm{~W}$ and in the band $915 \mathrm{MHz}$ to $921 \mathrm{MHz}$ with power levels up to $4 \mathrm{~W}$; Part 1: Technical requirements and methods of measurement, EN 302 208-1, 2016.

[14] PHYS370, Advanced Electromagnetism, Part 4: Waves and Boundaries, University of Liverpool, available at: http://pcwww.liv.ac.uk/ awolski/Teaching/Liverpool/PHYS370/Advan cedElectromagnetism,Part4.pdf [Accessed: March 2017].

[15] Smartrac, "DogBone, Excellent Global Performance even on DifficultTo-Tag Materials," Datasheet, 2017.

[16] Alien, "The Art and Science of UHF Passive Tag Design: and Selecting the Tag that is Best for Your Requirements," [Online]. Available at: http://www.alientechnology.com/wp-content/uploads/whitepaperAlien-Technology-The-Art-and-Science-of-Tag-Design-V1.0.pdf [Accessed: Dec. 2016].

[17] U. A. Bakshi and A. V. Bakashi, Antennas and Wave Propagation, Technical Publications Pune, India, 2009.

[18] R. S. Howard and H. D. Vaughan, NEETS (Navy Electricity and Electronic Training Series) Module 10 - Introduction to Wave Propagation, Transmission Lines, and Antennas (NAVEDTRA 14182). Naval Education and Training Center, US Navy, 1998.

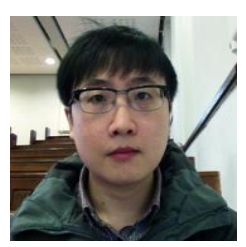

Shuai Yang (S'15) received the B. Eng. degree from the Opto-electronic Engineering Department, Changchun University of Science and Technology, China, in 2009, and the M.Sc. degree (with honors) in Photonic and Optical Engineering from the University of Nottingham, Nottingham, U.K., in 2011, and the M.Res. degree in Photonic Systems Development from the University of Cambridge, Cambridge, U.K., in 2013. He is currently a PhD student in the CPS group at the University of Cambridge. His current research focuses on RF power delivery for passive RFID and next generation battery-less sensors.

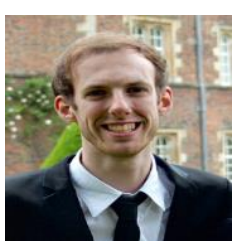

Nicolas Scirocco (S'17) Nicolas Scirocco is currently a $\mathrm{PhD}$ student in the CPS group at the University of Cambridge. Nicolas holds a Master's degree from Cambridge in integrated photonic and electronics systems. Prior to this, he studied physics and graduated at the top of his year from King's College London. His current research interests include RFID technology, electromagnetics and antenna theory. 


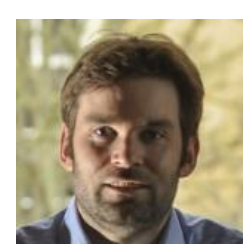

Michael Crisp (S'07-M'10) is currently a lecturer in Photonics and RF Systems in the Engineering Department at the University of Cambridge, Cambridge UK. He completed both his undergraduate studies and $\mathrm{PhD}$ in optical and RF systems, exploiting the benefits of optical fibres for the analog distribution of radio signals within buildings also at Cambridge University. Since his $\mathrm{PhD}$ he has remained in Cambridge working on radio frequency identification systems, for which he was awarded the Royal Academy of Engineering Young Entrepreneurs award in 2011 and co-founded a spin out company PervasID. His current research interests include RF systems for next generation battery-less sensors and analog applications of photonic systems.

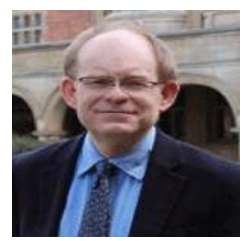

Richard V. Penty (M'00-SM'08) received the Ph.D. degree in engineering from the University of Cambridge, Cambridge, U.K., in 1989, for his research on optical fiber devices for signal processing applications. He was a Science and Engineering Research Council (SERC) Information Technology Fellow, at the University of Cambridge, working on all optical nonlinearities in waveguide devices. He is currently a Professor of Photonics at the University of Cambridge, having previously held academic posts at the Universities of Bath and Bristol. His research interests include high speed optical communications systems, photonic integration, optical switching and sensing systems. He has been the author of more than 750 refereed journal and conference papers. Prof. Penty is a Fellow of the Royal Academy of Engineering and of the IET. $\mathrm{He}$ is an Editor-in-Chief of the IET Optoelectronics Journal.

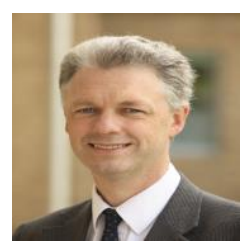

Ian H. White (S'82-M'83-SM'00-F'04) is currently Master of Jesus College, van Eck Professor of Engineering, Deputy Vice-Chancellor and Head of Photonics Research at the Department of Engineering, University of Cambridge. He gained his BA and $\mathrm{PhD}$ degrees from the University of Cambridge, in 1980 and 1984. He was then appointed a Research Fellow and Assistant Lecturer at the University of Cambridge before becoming Professor of Physics at the University of Bath in 1990. In 1996 he moved to the University of Bristol as Professor of Optical Communications and became Head of the Department of Electrical and Electronic Engineering in 1998, before returning to the University of Cambridge in October 2001. In 2005 he became Head of the School of Technology and subsequently Chair, leaving the School of Technology to take up the position of Pro-Vice-Chancellor for Institutional Affairs in 2010. Ian is a Fellow of the Royal Academy of Engineering and of the Institution of Electrical Engineers and the Institute of Electrical and Electronics Engineers. He is an Editor-in-Chief of Electronics Letters, has published in excess of 250 journal papers, and received the Aron Kressel Award from the Institute of Electrical and Electronics Engineers in 2011. He is a co-founder of Zinwave Ltd and PervasID Ltd. 